\title{
PLASMOCITOMA OVARIANO EM PORTADORA DE MIELOMA MÚLTIPLO: RELATO DE CASO
}

\author{
Bruno Rabelo BERNARDES ${ }^{1}$ \\ Mariana Moura Quintão SILVA ${ }^{2}$ \\ Flávio Assumpção Zambelli LOYOLA ${ }^{3}$ \\ Igor Dominick MICHALICK ${ }^{4}$ \\ Isabela Souza de PAULA ${ }^{5}$ \\ Seiji MIYATA ${ }^{6}$
}

1 - Médico residente de Cirurgia Geral do Hospital Luxemburgo/Instituto Mario Penna

(email: bruno-r-b@hotmail.com)

2 - Médico residente de Cirurgia Geral do Hospital Luxemburgo/Instituto Mario Penna

(email: mariquintao@gmail.com)

3 - Médico residente de Cirurgia Geral do Hospital Luxemburgo/Instituto Mario Penna

(email: flaviozambelli@gmail.com)

4 - Medico Cirurgião Geral e residente de Cirurgia Oncológica do Hospital Luxemburgo/Instituto Mario Penna

(email: igor.michalick@gmail.com)

5 - Médica Cirurgiã Geral e residente de Cirurgia de Cabeça e Pescoço do Hospital Luxemburgo/Instituto Mario Penna

(email: isabelaspaula@hotmail.com)

6- Médico Cirurgião Geral e Cirurgião Oncológico do Hospital Luxemburgo/Instituto Mario Penna

(email: seijisilvana@terra.com.br)

\section{Recebido em: 21/05/2014 - Aprovado em: 30/06/2014 - Disponibilizado em: 30/07/2014}

Resumo: A ocorrência de plasmocitomas extramedulares é incomum e pode ocorrer de forma isolada ou no contexto do mieloma múltiplo. Este artigo visa relatar um caso de plasmocitoma ovariano, dada a raridade deste achado e comparar com outros poucos casos descritos na literatura. Relata-se o caso de paciente do sexo feminino de 58 anos, com história de dor abdominal e em membro inferior esquerdo, cuja tomografia computadorizada evidenciou massa pélvica bilateral e lesão osteolítica em fêmur esquerdo. Foi submetida à laparotomia com ooforectomia esquerda e salpingoooforectomia direita. Imuno-histoquímica sugere mieloma múltiplo/plasmocitoma e linfoma plasmablástico. Exames evidenciaram imunoglobulina monoclonal IgG/kappa e mielograma confirmou o diagnóstico de mieloma múltiplo.Foram encontrados nove casos de plasmocitoma ovariano publicados na literatura, de pacientes com quadro de dor ou massa abdominal, submetidos a ressecção de tumor pélvico. Detectou-se imunoglobulina monoclonal em cinco destes casos, sugerindo mieloma múltiplo associado.O tratamento da paciente foi realizado com esquema CTP, diferente do relatado nos demais casos, com marcadores clínicos e laboratoriais evidenciando excelente resposta.

Palavras-chave: Plasmocitoma. Mieloma Múltiplo. Ovário. Dor abdominal. Ooforectomia.

\begin{abstract}
The occurrence of extramedullary plasmacytomas is uncommon and can happen in solitary form or in the context of multiple myeloma. This article aims to report a case of plasmacytoma of the ovary, given the rarity of this finding, and compare with a few other cases described in the literature. We report the case of a female patient of 58 years with a history of abdominal and left lower limb pain, whose CT scan showed bilateral pelvic mass and osteolytic lesion in the left femur. She underwent laparotomy with left oophorectomy and right salpingo- oophorectomy. Immunohistochemistry suggested multiple myeloma / plasmacytoma and plasmablastic lymphoma. Laboratory tests presented monoclonal immunoglobulin IgG / kappa and myelogram confirmed the diagnosis of multiple myeloma. Nine cases of ovarian plasmacytoma were found in the literature, that describes patients with pain or abdominal mass underwent resection of a pelvic tumor. Monoclonal immunoglobulin was detected in five of these cases, suggesting concomitant multiple myeloma. The treatment of the patient was performed with CTP scheme, different from the other reported cases, with clinical and laboratory markers showing excellent response.
\end{abstract}

Key words: Plasmacytoma. Multiple Myeloma. Ovary. Abdominal pain. Ooforectomy. 


\section{INTRODUÇÃO}

O Mieloma Múltiplo (MM) é uma neoplasia de células $B$, progressiva, caracterizada pela proliferação desregulada e clonal de plasmócitos na medula óssea. As consequências fisiopatológicas do avanço da doença incluem destruição óssea, falência renal, supressão da hematopoese e maior risco de infecções (RAJKUMAR 2014; FANNING, 2013; PAULA E SILVA et al, 2009).

O acometimento extramedular pelo MM, na forma de plasmocitomas, é incomum. Decorre da proliferação de plasmócitos geralmente em superfícies mucosas (RAJKUMAR 2014), preferencialmente no trato respiratório superior. Plasmocitomas podem se apresentar como lesões únicas e solitárias (Plasmocitoma solitário) na medula óssea ou em sítios extramedulares ou no contexto do MM (FANNING, 2013).

\section{DESCRIÇÃO DO CASO}

Paciente OFS, 58 anos, sexo feminino, com história de fratura de fêmur com correção cirúrgica em outro serviço em 2011, sem relato de investigação para possível causa secundária na época. No inicio de 2013 iniciou quadro de fraqueza e dor em membro inferior esquerdo e dor abdominal. Realizou tomografia computadorizada (TC) de abdome em julho de 2013 que evidenciou massa tumoral em cavidade pélvica comprometendo anexos bilateralmente, medindo $12 \times 7,3 \mathrm{~cm}$ em seus maiores eixos (FIGURA 1).

A paciente foi então encaminhada ao serviço de Cirurgia Oncológica do Hospital Luxemburgo, no qual foi avaliada em agosto de 2013. Ao exame apresentava lesão endurecida, em coxa esquerda proximal, de cerca de $15 \mathrm{~cm}$ de extensão, além de dor à palpação profunda em hipogástrio e fossas ilíacas, sem massas abdominais palpáveis.

Foi então indicada a realização de laparotomia exploradora. Ao inventário da cavidade evidenciou-se massa em ambos os ovários, de cerca de $5 \mathrm{~cm}$ de diâmetro cada.

FIGURA 1 - Massa pélvica em TC de abdome

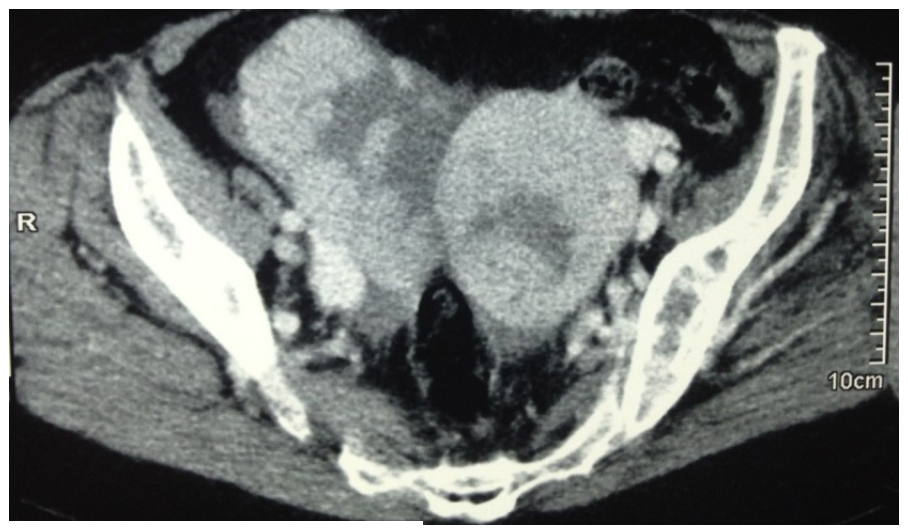

Fonte - Fotografia de TC de abdome obtida em nosso serviço 
Procedeu-se então com realização de ooforectomia esquerda e ooforectomia com salpingectomia direita. Paciente evoluiu bem e recebeu alta hospitalar no $2^{\circ}$ dia de pósoperatório.

O estudo anatomopatológico dos ovários revelou, em ambos, alterações indicativas de neoplasia pouco diferenciada constituída de células de aspecto linfoplasmo-histiocitóide atípicas. A imunohistoquímica sugeriu neoplasia maligna hematopoiética de alto grau, padrão plasmoblástico com expressão monotípica para cadeia leve de imunoglobulina kappa, interrogando-se Mieloma Múltiplo/ Plasmocitoma e linfoma plasmablástico

FIGURA 2 - Foto das lâminas com marcadores de imunohistoquimica
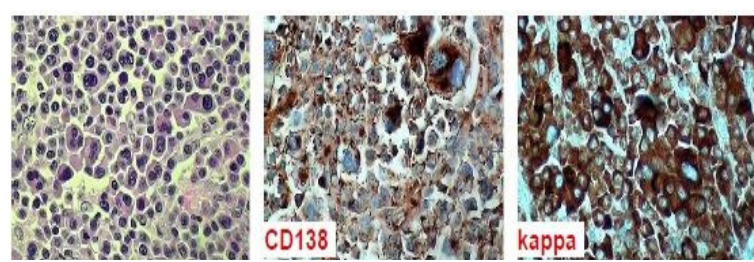

Fonte - Fotografia de exame obtida em nosso serviço

Paciente foi então encaminhada à Oncologia Clínica a qual deu seguimento à investigação diagnóstica. Exames evidenciaram anemia, cálcio e creatinina normais, avaliação urinária negativa para proteínas monoclonais, eletroforese de proteínas com pico monoclonal em beta2 e imunofixação sérica com padrão monoclonal IgG/kappa. TC de membro inferior esquerdo evidenciou lesão osteolítica em fêmur proximal (FIGURA 3) e TC de tórax mostrou seis nódulos pulmonares, bilaterais, com o maior medindo $5,3 \mathrm{~cm}$ e duas estruturas osteoblásticas nos corpos de $\mathrm{T} 8$ e $\mathrm{T} 10$ (FIGURA 4). Estudo radiológico de demais grupos ósseos sem alterações. Submetida à biópsia de medula óssea que evidenciou $13 \%$ de plasmócitos, confirmando-se finalmente o diagnóstico de Mieloma Múltiplo IgG/kappa.

Em outubro de 2013 foi então iniciado o tratamento com CTD (ciclofosfamida, talidomida, dexametasona), com grande melhora do quadro de dor em coxa esquerda e da anemia, além de queda do pico em beta2, o que indica excelente resposta ao tratamento. Atualmente encontra-se no $7^{\circ}$ ciclo de tratamento e está em protocolo para realização de transplante autólogo de medula óssea.

\section{DISCUSSÃO}

O Mieloma Múltiplo (MM) corresponde a 1 por cento de todos os cânceres e a pouco mais de 10 por cento das neoplasias malignas hematológicas nos Estados Unidos (FANNING, 2013). Estatística semelhante foi encontrada em trabalhos europeus. A idade média de surgimento do MM é de 66 anos, sendo rara em pessoas abaixo dos 50 anos de idade.

O MM consiste na proliferação de um clone único de plasmócito na medula óssea e, menos comumente, em sítios extramedulares, 
FIGURA 3 - Lesão osteolítica em fêmur esquerdo proximal em TC de bacia

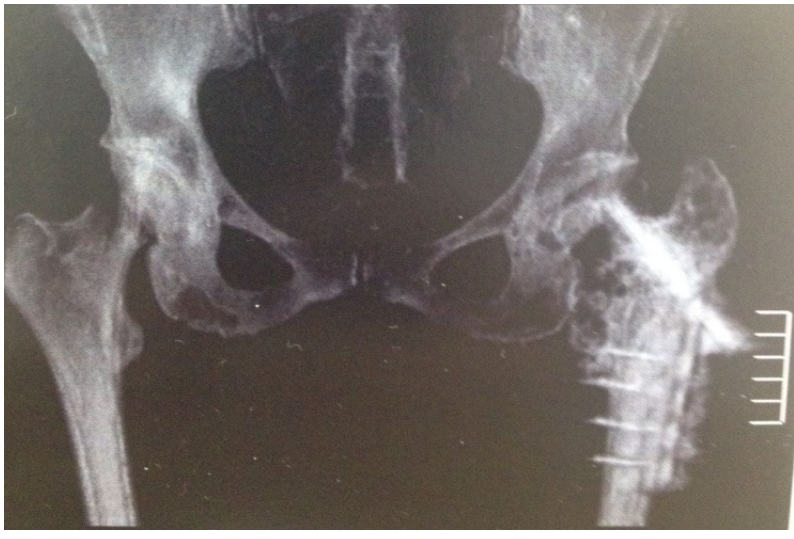

células estas que secretam imunoglobulina

Fonte - Fotografia de exame obtida em nosso serviço

Clinicamente pode cursar com dor óssea, fraturas patológicas, astenia, perda de peso, anemia, piora da função renal, hipercalcemia e infecções (RAJKUMAR, 2014; FANNING, 2013; PAULA E SILVA et al, 2009).

Plasmocitomas existem em três formas clínicas: Plasmocitoma medular solitário, Plasmocitoma extramedular solitário e Mieloma Múltiplo. Nas duas primeiras formas, são encontrados tumores únicos compostos por plasmócitos, sem outras evidências de MM, como infiltrado de plasmócitos maior que 5 por cento do total, anemia, hipercalcemia, aumento de escórias renais e elevação de níveis séricos ou urinários de imunoglobulina monoclonal (FANNING, 2013; SHAKUNTALA et al, 2013). Utiliza-se o termo "plasmablástico" quando são encontrados mais de 30 por cento

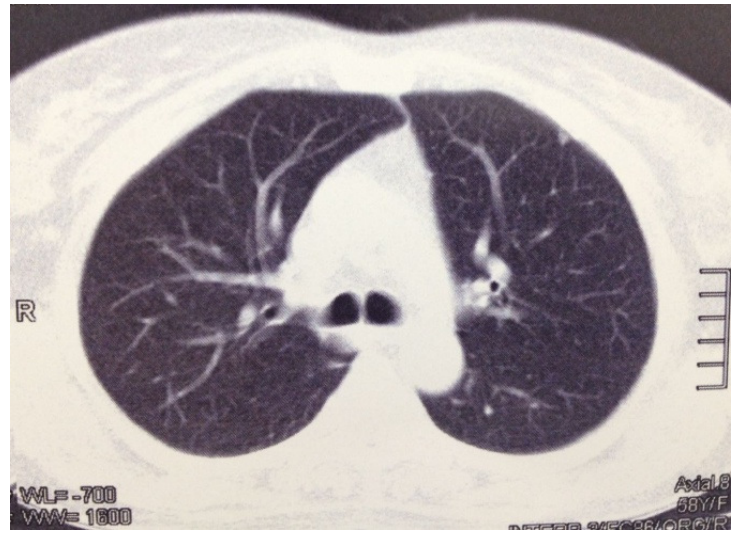

de blastos no estudo histológico do tumor (FANNING, 2013).

Plasmocitomas extramedulares (PEM) são encontrados em cerca de 7 por cento dos pacientes portadores de MM no momento do diagnóstico, como no caso relatado, e cerca de 6 por cento dos pacientes desenvolverão PEM após o diagnóstico (FANNING, 2013). Estes se desenvolvem, em cerca de $90 \%$ dos casos, no trato respiratório superior (SHAKUNTALA et al, 2013), e mais raramente em pele, pulmões, baço, fígado, trato gastrointestinal, rins, linfonodos, dentre outros tecidos moles, como ovários (RAJKUMAR, 2014; SHAKUNTALA et al, 2013). A paciente em questão apresentava comprovadamente plasmocitomas ovarianos e, possivelmente, plasmocitomas pulmonares, dois sítios raros de acometimento.

Em relação aos nove casos de plasmocitoma ovariano até então publicados na literatura, o primeiro caso data de 1930 e 
pouca informação foi disponibilizada pelo relato; o último caso publicado data de 2012. A idade de diagnóstico variou entre 12 e 63 anos, porém, sete deles ocorreram em maiores de 35 anos. Os sinais e sintomas que instigaram investigação adicional foram principalmente dor abdominal e/ou massa abdominal palpável ao exame físico - no caso de nossa paciente apenas dor estava presente. Ocorreu envolvimento bilateral dos ovários em um caso, assim como no nosso relato, unilateral direito em quatro casos, unilateral esquerdo em dois casos e unilateral não especificado em um caso. O diâmetro da massa ressecada foi igual ou superior a $12 \mathrm{~cm}$ em todos os casos descritos. Eletroforese de proteínas foi realizada em seis dos nove relatos, tendo sido identificada imunoglobulina monoclonal em cinco destes, sugerindo Mieloma Múltiplo associado.

Quanto ao tratamento adjuvante, este é descrito somente nos últimos dois casos, ambos publicados em 2012, tendo sido realizado em um deles com esquema quimioterápico DECP e o outro com carboplatina. No primeiro caso, relata-se haver MM associado, porém não constam dados a respeito do seguimento e resposta ao tratamento instituído. No segundo caso, descreve-se um caso PEM ovariano em sua forma isolada e há o relato de excelente resposta ao tratamento com carboplatina, com um seguimento de 24 meses livre de doença.
No caso da paciente aqui descrita, considerando a presença de PEM no contexto de MM, optou-se por terapia de indução com o esquema CTD (ciclofosfamida, talidomida, dexametasona) seguido de transplante autólogo de medula óssea (TAMO), esta ainda não realizada até o momento. Terapia de altas doses combinada com o TAMO proporciona uma resposta superior e maior sobrevida quando comparado com a quimioterapia padrão em pacientes com diagnóstico recente de MM (MORGAN et al, 2012).

\section{CONCLUSÃO}

Até o momento não há consenso sobre terapia adjuvante pós-operatória em plasmocitoma extramedular de ovário. Os dados relacionados a progressão e sobrevivência são muito escassos e são recolhidos a partir de relatos de casos. No caso descrito o tratamento instituído se baseou na doença de base, o Mieloma Múltiplo, e não especificamente na ocorrência de plasmocitomas extramedulares.

Em todos os casos descritos foi realizada a ressecção cirúrgica do plasmocitoma ovariano e em alguns seguiu-se com quimioterapia adjuvante. No caso da paciente em questão ressecou-se a massa e, firmado o diagnóstico de MM, seguiu-se com terapia com CTP, produzindo bons resultados até o momento. Nota-se, porém, a necessidade 
de mais estudos para definição terapêutica nos

casos de plasmocitoma ovariano, tanto em sua

\section{REFERÊNCIAS}

ALEXIOU C, KAU RJ, DIETZFELBINGER H, KREMER M, SPIESS JC AND ARNOLD W. Extramedullary plasmacytoma: tumor occurrence and therapeutic concepts. Cancer. 1999; 85:2305-14

ANDZE G, PAGBE JJ, TCHOKOTEU

$\mathrm{PF}$ et al. Le plasmacytome solitaire extraosseux ovarien J Chir. 1993; 130 $137-40$

BAMBIRRA EA, MIRANDA D, MAGALHAES GMC. Plasma cell myeloma simulating Krukenberg's tumor South Med J. 1982; 75 511-2

COOK HT, BOYLSTON AW.

Plasmacytoma of the ovary Gynecol

Oncol. 1988; 29:378-81

EMERY JD, KENNEDY AW, TUBBS

RR, CASTELLANI WJ, HUSSEIN MA.

Plasmacytoma of the ovary: a case report and literaturereview Gynecol Oncol. 1999; 73(1) 151-4

FANNING SR. Extramedullary plasmacytoma. Medscape [Internet]. [atualizado em 2013 Jun 12; citado em 2014 Abr 30]. Disponível em: http://emedicine.medscape.com/article/20 7233-overview

HAUTZER NW. Primary plasmacytoma of ovary. Gynecol Oncol. 1984; 18:115-8

MORGAN GJ, DAVIES FE, GREGORY WM, BELL SE, SZUBERT AJ, COY NN,COOK G, FEYLER S, JOHNSON PRE, RUDIN C, DRAYSON MT, OWEN RG, ROSS FM, RUSSELL NH, JACKSON GH, CHILD JA. forma solitária como na associada ao Mieloma Múltiplo.

Cyclophosphamide, thalidomide, and dexamethasone as induction therapy for newly diagnosed multiple myeloma patients destined for autologous stem-cell transplantation: MRC Myeloma IX randomized trial results. Haematologica. 2012 March; 97(3): 442-450. doi: 10.3324/haematol.2011.043372

PAULA E SILVA RO, BRANDÃO KAM, PINTO PVM, FARIA RMD, CLEMENTINO NCD, SILVA CMF, LOPES AF. Mieloma múltiplo: características clínicas e laboratoriais ao diagnóstico e estudo prognóstico. Rev. Bras. Hematol. Hemoter. 2009;31(2):6368

RAJKUMAR SV. Clinical features, laboratory manifestations and diagnosis of multiple myeloma. UpToDate [Internet]. [atualizadao em 2014 Abr 04; citado em 2014 Abr 30]. Disponível em: http://www.uptodate.com/contents/clinical -features-laboratory-manifestations-anddiagnosis-of-multiple-myeloma

RAJKUMAR SV. Pathobiology of multiple myeloma. UpToDate [Internet]. [atualizado em 2014 Fev 14; citado em 2014 Abr 30. Disponível em: http://www.uptodate.com/contents/pathobi ology-of-multiple-

$\underline{\text { myeloma?source }=\text { search_result\&search }=\mathrm{P}}$ athobiology+of+multiple+myeloma\&sele ctedTitle $=1 \sim 150$

SHAKUNTALA PN, PRAVEEN SR, SHANKARANAND B, RAJSHEKAR K, UMADEVI K, BAFNA UD. A rare case of plasmacytoma of the ovary: a case report and literature review. Ecancermedicalscience. 2013, 7:288 
TALERMAN A. Nonspecific tumors of the ovary, including mesenchymal tumors and malignant lymphoma Blaustein's Pathology of the Female Genital Tract 3rd edn, ed RJ Kurman. 1987; 738 pp

VOEGT H. Extramedullary plasmacytoma. Virchows Arch Pathol

Anat. 1938; 302:497-508

ZHONG YP, ZHANG JJ, HUANG XN. Multiple myeloma with rupture of ovarian plasmacytoma. Chin Med J (Engl). 2012; 125(16) 2948-50 